doi: $10.15407 /$ ujpe62.03.0256

R.M. PELESHCHAK, M.YA. SENETA

Ivan Franko State Pedagogical University of Drohobych

(24, Ivan Franko Str., Drohobych 82100, Lviv region, Ukraine; e-mail: marsen18@i.ua)

\title{
DISPERSION LAW AND THE DEPENDENCE OF THE SURFACE ACOUSTIC MODE WIDTH PACS 81.07.Bc, 66.30.Lw ON THE CONCENTRATION OF ADSORBED ATOMS
}

\begin{abstract}
The dispersion law for elastic surface acoustic waves and the dependence of the surface acoustic mode width on the concentration of adsorbed atoms have been found. The calculations are carried out in the long-wave approximation for the interaction between the adatoms with regard for image forces and the non-local elastic interaction between the adsorbed and matrix atoms.

Ke yw ords: deformation potential, adatoms, non-local elastic interaction, image forces, surface acoustic mode width.
\end{abstract}

\section{Introduction}

The method of surface acoustic waves (SAWs) has been widely used recently to study the dynamic parameters (dynamic conductivity, charge carrier mobility, and concentration) of two-dimensional electron layers in $\mathrm{Al}_{1-x} \mathrm{Ga}_{x}$ As heterostructures [1-3], which demonstrate piezoelectric properties. Nanoheterosystems with strained $\mathrm{GaAs} / \mathrm{In}_{1-x} \mathrm{Ga}_{x} \mathrm{As} / \mathrm{GaAs}$ [4], $\quad \mathrm{Cd}_{1-x} \mathrm{Zn}_{x} \mathrm{Te} / \mathrm{CdTe} / \mathrm{Cd}_{1-x} \mathrm{Zn}_{x} \mathrm{Te}[5,6]$, and $\mathrm{CdTe} / \mathrm{HgTe}$ [6] layers are characterized by both non-uniform deformation and non-uniform piezoelectric fields. An SAW generates an alternating electric field and a dynamic deformation field. The latter creates irregularities on the semiconductor surface [7], which are responsible for the emergence of regions with surface electron states at the semiconductor interface. Those regions are characterized by different physical properties. They are separated by an energy gap, the width of which is determined by the irregularity height. The latter can depend on both the concentration of adsorbed atoms and the deformation potential magnitude [8]. Furthermore, the interaction of the alternating electric field with two-dimensional electrons [3] and the interaction of the dynamic deformation field with adsorbed atoms result in the renormalization of the SAW velocity and damping.

The authors of work [9] studied the influence of interaction between the surface elastic Rayleigh wave and the electron-hole plasma in a two-dimensional

(C) R.M. PELESHCHAK, M.YA. SENETA, 2017 semimetal (HgTe) - this interaction is driven by two mechanisms: the deformation potential and the piezoelectric effect - on the dispersion law and the damping of Rayleigh waves. The influence of a structurally distorted isotropic surface layer that was grown up on the surface of isotropic solid on the dispersion law and the reciprocal damping length of Rayleigh waves was analyzed in work [10].

Since SAWs can be one of the sources that are responsible for long-range effects stimulating the formation of nanoclusters beyond the laser-irradiated region on the crystal surface [11], the researches of the processes giving rise to the damping of surface elastic acoustic waves on a single-crystalline substrate surface with defects are challenging. The aim of this work was to calculate the dispersion law and the width of the surface elastic acoustic modes as the functions of the concentration of adsorbed atoms taking into account the non-local elastic interaction of adatoms with the self-consistent quasi-Rayleigh acoustic wave, as well as image forces.

\section{Model of Adatom Interaction with a Surface Acoustic Wave}

Let a semiconductor surface coincide with the plane $z=0$ (the axis $z$ is directed into the single crystal depth). This surface is bombarded with a flux of atoms obtained in the course of the molecular beam epitaxy process. The adsorbed atoms are considered as defects on the surface (elastic surface inclusions). Owing to the deformation potential and the local renormalization of surface energy, the adsorbed ISSN 2071-0194. Ukr. J. Phys. 2017. Vol. 62, No. 3 
atoms and the surface elastic quasi-Rayleigh acoustic wave together non-uniformly deform the nearsurface layer of the thickness $a$ ( $a$ is the lattice constant along the axis $z$ ). In its turn, the arising selfconsistent non-uniform deformation, acting through the deformation potential, redistributes the adsorbed atoms over the surface, i.e. it induces an additional deformation-diffusive flux of adatoms $[12,13]$ The influence of adsorbed atoms is reduced to the change of boundary conditions for the strain tensor $\sigma_{i j}$ at the surface $z=0$.

The displacement vector of a point in the medium, $\mathbf{u}(\mathbf{r}, t)$, satisfies the equation [14]

$$
\frac{\partial^{2} \mathbf{u}}{\partial t^{2}}=c_{t}^{2} \Delta_{\mathbf{r}} \mathbf{u}+\left(c_{l}^{2}-c_{t}^{2}\right) \overrightarrow{\operatorname{grad}}(\operatorname{div} \mathbf{u}) .
$$

The solution of this equation for the surface Rayleigh wave propagating along the axis $x$ is sought in the form

$u_{x}(x, z)=-i q A e^{i q x-i \omega t-k_{l} z}-i k_{t} B e^{i q x-i \omega t-k_{t} z}$,

$u_{z}(x, z)=k_{l} A e^{i q x-i \omega t-k_{l} z}+q B e^{i q x-i \omega t-k_{t} z}$,

where $k_{l, t}^{2}=q^{2}-\frac{\omega^{2}}{c_{l, t}^{2}}$; and $A$ and $B$ are the SAW amplitudes.

The direction $x$ on the crystal surface is determined by elastic anisotropy. On an isotropic surface, it is determined either by an external action that induces the elastic anisotropy or owing to a spontaneous symmetry breaking in the defect-deformation system, similarly to work [15].

The strain $\varepsilon$ at the semiconductor surface $(z=0)$ is defined in terms of components of the displacement vector, by using the relation

$\varepsilon(x, t)=\frac{\partial u_{x}}{\partial x}+\frac{\partial u_{z}}{\partial z}=\frac{\omega^{2}}{c_{l}^{2}} A e^{i q x-i \omega t}$.

The spatially non-uniform surface strain $\varepsilon(x, t)$ stimulates an inhomogeneous redistribution of adatoms $N_{d}(x, t)$ :

$N_{d}(x, t)=N_{d 0}+N_{d 1}(x, t)=N_{d 0}+N_{d 1}(q) e^{i q x-i \omega t}$,

where $N_{d 0}$ is the spatially homogeneous component, and $N_{d 1}(q)$ the periodic perturbation amplitude $\left(N_{d 1} \ll N_{d 0}\right)$. In the non-local Hooke's law approximation $[12,16,17]$, the energy of interaction of an

ISSN 2071-0194. Ukr. J. Phys. 2017. Vol. 62, No. 3 adsorbed atom with atoms of the matrix, $W_{d a}$, is determined as follows:

$W_{d a}(x)=-\int \lambda\left(\left|x^{\prime}-x\right|\right) \varepsilon\left(x^{\prime}\right) \Delta \Omega_{d} d x^{\prime}$,

where $\lambda$ is the operator of elastic moduli [16], and $\Delta \Omega_{d}$ a variation of the crystal volume induced by one adsorbed atom.

Let us introduce the variable $\tau=x^{\prime}-x$ and expand $\varepsilon\left(x^{\prime}\right)$ in a Taylor series in $\tau$ :

$W_{d a}^{\mathrm{int}}(x)=-\int \lambda(|\tau|) \varepsilon(x+\tau) \Delta \Omega_{d} d \tau=$

$=-\int \lambda(|\tau|)\left(\varepsilon(x)+\frac{\partial^{2} \varepsilon(x)}{\partial x^{2}} \frac{\tau^{2}}{2}\right) \Delta \Omega_{d} d \tau=$

$=-K_{d} \varepsilon(x) \Delta \Omega_{d}-K_{d} \frac{\partial^{2} \varepsilon(x)}{\partial x^{2}} l_{d}^{2} \Delta \Omega_{d}$,

where $K_{d}=\int \lambda(|\tau|) d \tau \equiv K$ is the elastic modulus, and

$l_{d}^{2}=\frac{\int \lambda(\tau) \tau^{2} d \tau}{2 \int \lambda(|\tau|) d \tau}$

is the average squared characteristic distance of the interaction of an adatom with atoms of the matrix.

The elastic fields created by adsorbed atoms shift atoms in vicinities of other adatoms and create forces acting on them, which results in their elastic interaction. The energy of this interaction decreases, by following the power law. This energy is rather substantial if adatoms strongly deform the crystal lattice. In isotropic objects, the energy of elastic interaction between defects equals zero. Along with the elastic interaction of adsorbed atoms, which decreases, by following the power law, as the distance between the adatoms grows, there exists an interaction that smoothly changes at distances of an order of the crystal size. This interaction is associated with image forces applied to the crystal surface. The corresponding energy $W_{d d}^{\text {int }}$ of interaction between an adsorbed atom located at $r^{\prime}$ and other adatoms distributed with the concentration $N_{d}(x)$ is practically independent of the adatom position $r^{\prime}$ and can be determined, by using the formula [17]

$W_{d d}^{\mathrm{int}}(x)=-\frac{2}{3} \frac{1-2 \nu}{K(1-\nu) a} \theta_{s}^{2} N_{d}(x)$,

where $\nu$ is Poisson's ratio, and $\theta_{s}=K \Delta \Omega_{d}$ is the surface deformation potential. 
The elastic field that arises in the matrix implanted with adatoms acts on the adatom with the force

$F=-\frac{\partial\left(W_{d d}^{\mathrm{int}}(x)+W_{d a}^{\mathrm{int}}(x)\right)}{\partial x}=\frac{2}{3} \frac{1-2 \nu}{K(1-\nu) a} \times$

$\times \theta_{s}^{2} \frac{\partial N_{d}(x)}{\partial x}+\theta_{s} \frac{\partial \varepsilon(x, t)}{\partial x}+\theta_{s} l_{d a}^{2} \frac{\partial^{3} \varepsilon(x, t)}{\partial x^{3}}$.

Besides the ordinary diffusion flux $\left(-D_{d} \frac{\partial N_{d}(x)}{\partial x}\right)$, this force induces an additional deformation flux of adatoms. The latter results from the strain, $\frac{\partial \varepsilon(x, t)}{\partial x}$, and defect concentration, $\frac{\partial N_{d}(x)}{\partial x}$, gradients.

The analysis of formula (9) demonstrates that the concentration gradient $\left(\theta_{s}^{2} \frac{\partial N_{d}(x)}{\partial x}\right)$ creates a component of the deformation flux. Unlike the ordinary diffusion flux, this component is directed in the direction of the adatom concentration growth (the first term). In addition, the adatoms that are stretching centers $\left(\Delta \Omega_{d}>0\right)$ move into a region that undergoes a relative stretching, whereas the adatoms that are squeezing centers $\left(\Delta \Omega_{d}<0\right)$ move into a region undergoing a relative squeezing (the second term). Under the action of force (9), the adatoms in the elastic field obtain the velocity

$v=\mu F=\frac{2}{3} \frac{1-2 \nu}{K(1-\nu)} \frac{D_{d} \theta_{s}^{2}}{k_{\mathrm{B}} T a} \frac{\partial N_{d}(x)}{\partial x}+$

$+\frac{D_{d} \theta_{s}}{k_{\mathrm{B}} T} \frac{\partial \varepsilon(x, t)}{\partial x}+\frac{D_{d} \theta_{s}}{k_{\mathrm{B}} T} l_{d a}^{2} \frac{\partial^{3} \varepsilon(x, t)}{\partial x^{3}}$,

where $D_{d}$ is the diffusion coefficient for the adatom, $T$ the temperature, $k_{\mathrm{B}}$ the Boltzmann constant, and the adatom mobility $\mu$ is determined using the Einstein relation.

In view of Eq. (10) and the continuity equation

$\operatorname{div} \mathbf{j}=-\frac{\partial N_{d}(x, t)}{\partial t}$

the flux of implanted adatoms equals

$$
\begin{aligned}
& j=-D_{d} \frac{\partial N_{d}(x, t)}{\partial x}+\frac{D_{d} \theta_{s}}{k_{\mathrm{B}} T} N_{d}(x, t) \times \\
& \times \frac{\partial}{\partial x}\left(\frac{2}{3} \frac{1-2 \nu}{K(1-\nu) a} \theta_{s} N_{d}(x, t)+\right. \\
& \left.+\varepsilon(x, t)+l_{d a}^{2} \frac{\partial^{2} \varepsilon(x, t)}{\partial x^{2}}\right),
\end{aligned}
$$

and the equation for the adatom concentration reads

$$
\begin{aligned}
& \frac{\partial N_{d}(x, t)}{\partial t}=D_{d} \frac{\partial^{2} N_{d}(x, t)}{\partial x^{2}}- \\
& -\frac{D_{d} \theta_{s}}{k_{\mathrm{B}} T} \frac{\partial}{\partial x}\left(N _ { d } ( x , t ) \frac { \partial } { \partial x } \left(\frac{2}{3} \frac{1-2 \nu}{K(1-\nu) a} \theta_{s} N_{d}(x, t)+\right.\right. \\
& \left.\left.+\varepsilon(x, t)+l_{d a}^{2} \frac{\partial^{2} \varepsilon(x, t)}{\partial x^{2}}\right)\right) .
\end{aligned}
$$

The first term in Eq. (12) describes the ordinary gradient-driven concentration diffusion, whereas the second one is a qualitatively new diffusion effect, "the flux of deformation drawing-in". The latter is governed by both image forces and the deformation gradient [18], on the one hand, and the non-local interaction of adatoms with surface atoms [19], on the other hand.

In the linear approximation with regard for the conditions $N_{d 1} \ll N_{d 0}$ and formula (5), Eq. (12) looks like

$\left(-i \omega+D_{d}\left(1-\frac{2}{3} \frac{1-2 \nu}{K(1-\nu) a} \frac{\theta_{d}^{2}}{k_{\mathrm{B}} T} N_{d 0}\right) q^{2}\right) N_{d 1}(q)=$

$=\frac{D_{d} \theta_{d}}{k_{\mathrm{B}} T} N_{d 0} \varepsilon(q) q^{2}\left(1-l_{d}^{2} q^{2}\right)$.

This equation is used to obtain an expression for the amplitude of the surface adatom concentration $N_{d 1}(q)$.

The spatially non-uniform distribution of adatoms modulates the surface energy

$F(x)=F_{0}+\frac{\partial F}{\partial N_{d 1}} N_{d 1}(x)$,

which results in the appearance of the lateral mechanical stress

$\sigma_{x z}=\frac{\partial F(N(x))}{\partial x}$,

which is compensated by the shift stress in the medium [14].

The boundary condition reflects a balance of lateral stresses:

$\left.\mu\left(\frac{\partial u_{x}}{\partial z}+\frac{\partial u_{z}}{\partial x}\right)\right|_{z=0}=\left(\frac{\partial F}{\partial N_{d 1}}\right) \frac{\partial N_{d 1}(x)}{\partial x}$,

where $\mu$ is the shear modulus of the medium. The coefficient $\frac{\partial F}{\partial N_{d 1}}$ is considered to be a given phenomenological parameter.

ISSN 2071-0194. Ukr. J. Phys. 2017. Vol. 62, No. 3 
Furthermore, owing to the interaction between the adatoms and the semiconductor atoms, there arises a normal mechanical stress on the semiconductor surface, so that the boundary condition looks like

$$
\left.\left(\frac{\partial u_{z}}{\partial z}+(1-2 \beta) \frac{\partial u_{x}}{\partial x}\right)\right|_{z=0}=\frac{\theta_{d} N_{d 1}(x)}{\rho c_{l}^{2} a},
$$

where $a$ is the crystal lattice constant at the semiconductor surface, $\beta=c_{t}^{2} / c_{l}^{2}$, and $\rho$ is the crystal density.

\section{Dispersion Equation} and the Width of the Surface Elastic Acoustic Mode Interacting with Adsorbed Atoms

In order to derive the dispersion equation, let us substitute Eq. (13) into Eqs. (14) and (15) with regard for Eqs. (2), (3), (4), and (5). As a result, we obtain a system of two linear equations for the amplitudes $A$ and $B$. The condition that its solutions must be non-trivial gives rise to the following dispersion equation for the surface acoustic wave interacting with adsorbed atoms:

$$
\begin{aligned}
& \left(q^{2}+k_{t}^{2}\right)^{2}-4 q^{2} k_{l} k_{t}=-\frac{2}{\beta} \frac{\omega^{2}}{c_{l}^{2}} \frac{\theta_{d} N_{d 0}}{k_{\mathrm{B}} T \rho c_{l}^{2}} \times \\
& \times \frac{D_{d} q^{2}}{-i \omega+D_{d}\left(1-\frac{2}{3} \frac{1-2 \nu}{K(1-\nu) a} \frac{\theta_{d}^{2}}{k_{\mathrm{B}} T} N_{d 0}\right) q^{2}} \times \\
& \times\left(1-l_{d}^{2} q^{2}\right)\left(q^{2} k_{t} \frac{\partial F}{\partial N_{d 1}}+\left(q^{2}+k_{t}^{2}\right) \frac{\theta_{d}}{2 a}\right) .
\end{aligned}
$$

The left-hand side of this equation coincides with the Rayleigh determinant, with the zero value of the latter determining the dispersion law for a surface Rayleigh acoustic wave in the absence of adsorbed atoms [14]. The right-hand side of Eq. (16) renormalizes the dispersion equation for the Rayleigh acoustic wave owing to the force action $\left(\sim \theta_{d}\right)$ of adsorbed atoms that deform the near-surface layer of the crystal lattice. Substituting $\omega=c_{t} q \xi$ into Eq. (16), we obtain

$$
\begin{aligned}
& \left(2-\xi^{2}\right)^{2}-4 \sqrt{1-\xi^{2}} \sqrt{1-\frac{c_{t}^{2}}{c_{l}^{2}} \xi^{2}}=-\frac{2 \xi^{2} \theta_{d} N_{d 0}}{k_{\mathrm{B}} T \rho c_{l}^{2}} \times \\
& \times \frac{D_{d} q\left(D_{d}\left(1-\frac{2}{3} \frac{1-2 \nu}{K(1-\nu) a} \frac{\theta_{d}^{2}}{k_{\mathrm{B}} T} N_{d 0}\right) q+i c_{t} \xi\right)}{\left(D_{d}\left(1-\frac{2}{3} \frac{1-2 \nu}{K(1-\nu) a} \frac{\theta_{d}^{2}}{k_{\mathrm{B}} T} N_{d 0}\right)\right)^{2} q^{2}+c_{t}^{2} \xi^{2}} \times \\
& \times\left(1-l_{d}^{2} q^{2}\right)\left(q \sqrt{1-\xi^{2}} \frac{\partial F}{\partial N_{d 1}}+\left(2-\xi^{2}\right) \frac{\theta_{d}}{2 a}\right) .
\end{aligned}
$$

Expression (17) has the real and imaginary parts, which ultimately determine a correction to the dispersion law for the Rayleigh wave and its damping, respectively. The presence of the factor $q$ in the numerator of Eq. (17) makes it possible to solve this equations in the long-wave region $(q a \ll 1)$ by the iteration procedure.

Let us designate the left-hand side of Eq. (17) as a function $f(\xi)$ and expand it in the Taylor series in a vicinity of the point $\xi_{0}$, which is a solution of the equation $f\left(\xi_{0}\right)=0$ :

$f\left(\xi_{0}+\delta \xi\right) \approx f\left(\xi_{0}\right)+f^{\prime}\left(\xi_{0}\right) \delta \xi$.

Then the correction $\delta \xi$ is determined by the righthand side of Eq. (17), in which the substitution $\xi \rightarrow$ $\rightarrow \xi_{0}$ was made:

$$
\begin{aligned}
& \delta \xi=-\frac{1}{f^{\prime}\left(\xi_{0}\right)} \frac{2 \xi_{0}^{2} \theta_{d} N_{d 0}}{k_{\mathrm{B}} T \rho c_{l}^{2}} \times \\
& \times \frac{D_{d}^{2} q^{2}\left(1-\frac{2}{3} \frac{1-2 \nu}{K(1-\nu) a} \frac{\theta_{d}^{2}}{k_{\mathrm{B}} T} N_{d 0}\right)}{\left(D_{d}\left(1-\frac{2}{3} \frac{1-2 \nu}{K(1-\nu) a} \frac{\theta_{d}^{2}}{k_{\mathrm{B}} T} N_{d 0}\right)\right)^{2} q^{2}+c_{t}^{2} \xi_{0}^{2}} \times \\
& \times\left(1-l_{d}^{2} q^{2}\right)\left(q \sqrt{1-\xi_{0}^{2}} \frac{\partial F}{\partial N_{d 1}}+\left(2-\xi_{0}^{2}\right) \frac{\theta_{d}}{2 a}\right)- \\
& -i \frac{1}{f^{\prime}\left(\xi_{0}\right)} \frac{2 c_{t} \xi_{0}^{3} \theta_{d} N_{d 0}}{k_{\mathrm{B}} T \rho c_{l}^{2}} \times \\
& \times \frac{D_{d} q}{\left(D_{d}\left(1-\frac{2}{3} \frac{1-2 \nu}{K(1-\nu) a} \frac{\theta_{d}^{2}}{k_{\mathrm{B}} T} N_{d 0}\right)\right)^{2} q^{2}+c_{t}^{2} \xi_{0}^{2}} \times \\
& \times\left(1-l_{d}^{2} q^{2}\right)\left(q \sqrt{1-\xi_{0}^{2}} \frac{\partial F}{\partial N_{d 1}}+\left(2-\xi_{0}^{2}\right) \frac{\theta_{d}}{2 a}\right) .
\end{aligned}
$$

A numerical analysis testifies that $f^{\prime}\left(\xi_{0}\right)>0$ in the whole domain of the variable $\xi_{0}$.

Extracting the real and imaginary parts of Eq. (19) and taking the relation $\omega=c_{t} q \xi_{0}+c_{t} q \delta \xi$ into account, we obtain the dispersion law $\omega^{\prime}(q)$ for the surface elastic acoustic wave in the form

$$
\begin{aligned}
& \omega^{\prime}(q)=c_{t} q \xi_{0}\left(1-\frac{1}{f^{\prime}\left(\xi_{0}\right)} \frac{2 \xi_{0} \theta_{d} N_{d 0}}{k_{\mathrm{B}} T \rho c_{l}^{2}} \times\right. \\
& \times \frac{D_{d}^{2} q^{2}\left(1-\frac{2}{3} \frac{1-2 \nu}{K(1-\nu) a} \frac{\theta_{d}^{2}}{k_{\mathrm{B}} T} N_{d 0}\right)}{\left(D_{d}\left(1-\frac{2}{3} \frac{1-2 \nu}{K(1-\nu) a} \frac{\theta_{d}^{2}}{k_{\mathrm{B}} T} N_{d 0}\right)\right)^{2} q^{2}+c_{t}^{2} \xi_{0}^{2}} \times \\
& \left.\times\left(1-l_{d}^{2} q^{2}\right)\left(q \sqrt{1-\xi_{0}^{2}} \frac{\partial F}{\partial N_{d 1}}+\left(2-\xi_{0}^{2}\right) \frac{\theta_{d}}{2 a}\right)\right),
\end{aligned}
$$


and the following expression for its width $\omega^{\prime \prime}(q)$, which is associated with the interaction between the adsorbed atoms and the self-consistent quasiRayleigh wave:

$$
\begin{aligned}
& \omega^{\prime \prime}(q)=-c_{t}^{2} \frac{1}{f^{\prime}\left(\xi_{0}\right)} \frac{2 \xi_{0}^{3} \theta_{d} N_{d 0}}{k_{\mathrm{B}} T \rho c_{l}^{2}} \times \\
& \times \frac{D_{d} q^{2}}{\left(D_{d}\left(1-\frac{2}{3} \frac{1-2 \nu}{K(1-\nu) a} \frac{\theta_{d}^{2}}{k_{\mathrm{B}} T} N_{d 0}\right)\right)^{2} q^{2}+c_{t}^{2} \xi_{0}^{2}} \times \\
& \times\left(1-l_{d}^{2} q^{2}\right)\left(q \sqrt{1-\xi_{0}^{2}} \frac{\partial F}{\partial N_{d 1}}+\left(2-\xi_{0}^{2}\right) \frac{\theta_{d}}{2 a}\right) .
\end{aligned}
$$

The obtained formulas make allowance for both the non-local elastic interaction of an implanted impurity with the matrix atoms [16] and image forces [17].

\section{Dispersion Law and the Width of the Surface Elastic Acoustic Mode. Numerical Calculation and Analysis of Their Dependences on the Concentration of Adsorbed Atoms}

The dispersion law $\omega^{\prime}(q)=\operatorname{Re} \omega(q)$ for the surface elastic acoustic wave and the width $\omega^{\prime \prime}(q)=$ $=\operatorname{Im} \omega(q)$ of the acoustic mode were calculated for the GaAs (001) semiconductor with the surface concentrations of adsorbed atoms $N_{d 0}=3 \times 10^{12}$ and $3 \times 10^{13} \mathrm{~cm}^{-2}$. The values of other parameters were as follows: $l_{d}=2.9 \mathrm{~nm}, a=0.565 \mathrm{~nm}$, $c_{l}=4400 \mathrm{~m} / \mathrm{s}, c_{t}=2475 \mathrm{~m} / \mathrm{s}, \rho=5320 \mathrm{~kg} / \mathrm{m}^{3}$, $D_{d}=5 \times 10^{-2} \mathrm{~cm}^{2} / \mathrm{s}, \theta_{d}=10 \mathrm{eV}, \partial F / \partial N_{d 1}=0.1 \mathrm{eV}$,

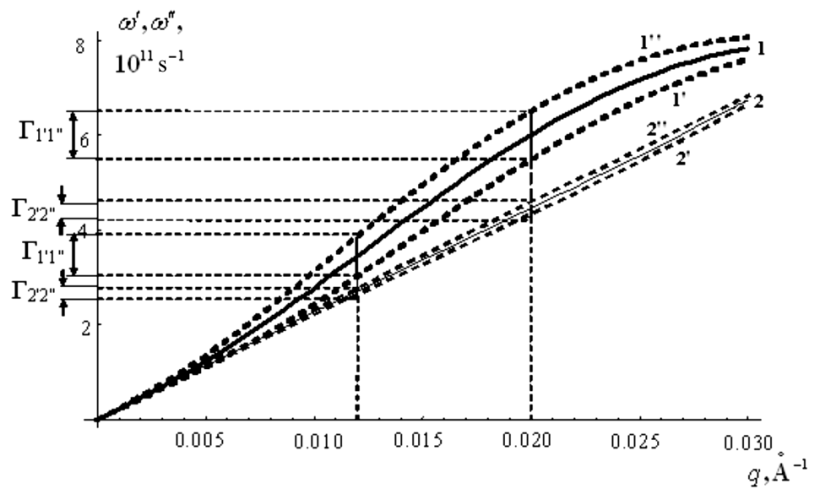

Fig. 1. Dispersion law and the phonon mode width for the surface elastic acoustic wave interacting with adsorbed atoms. Image forces and the non-local character of the interaction between the adsorbed atom and the matrix atoms $\left(l_{d} \neq 0\right)$ are taken into consideration and $T=100 \mathrm{~K}[13]$. The value of characteristic length of the interaction between an adatom and lattice atoms, $l_{d}$, was determined from the minimum condition for the free energy of the crystal with adsorbed atoms [12].

Figure 1 illustrates the results of calculations carried out for the dispersion law $\omega^{\prime}(q)$ of the surface elastic acoustic mode (curves 1 and 2) and for the dependence $\omega^{\prime \prime}(q)$ of its width on the absolute value of the wave vector $q$ (curves $1^{\prime}, 1^{\prime \prime}$ and $2^{\prime}, 2^{\prime \prime}$ ). The latter dependence arises due to the account for the interaction between the adsorbed atoms and the selfconsistent quasi-Rayleigh wave, and it was calculated making allowance for image forces. The calculations were performed for two indicated values of the adsorbed atom concentration $N_{d 0}$.

One can see that the dependences $\omega^{\prime}(q)$ and $\omega^{\prime \prime}(q)$ are non-linear in the interval $0 \leq q<\frac{1}{l_{d}}$. If $q \rightarrow 0$, the width of the surface acoustic mode $\omega^{\prime \prime}(q)$ tends to zero, whereas the dispersion curve $\omega^{\prime}(q)$ asymptotically approaches the dispersion curve $\omega(q)=c_{t} \xi_{0} q$ for the surface Rayleigh wave. It has to be noted that, at $q=\frac{1}{l_{d}}$, the length of the surface acoustic wave is identical to the characteristic length of the interaction between an adatom and lattice atoms. As one can see from Fig. 1, the width of the acoustic phonon mode grows with the concentration of adsorbed atoms. In particular, at $q=0.012 \AA$, the energy width for the surface acoustic mode $\hbar \omega^{\prime \prime}(q)$ amounts approximately to $\Gamma_{2^{\prime} 2^{\prime \prime}}=8.9 \mu \mathrm{eV}$, if the adatom concentration $N_{d 0}=3 \times 10^{12} \mathrm{~cm}^{-2}$, and to $\Gamma_{1^{\prime} 1^{\prime \prime}}=55 \mu \mathrm{eV}$, if $N_{d 0}=3 \times 10^{13} \mathrm{~cm}^{-2}$. In the shortwave interval for the surface elastic acoustic waves (at $q=0.02 \AA$ ), the energy widths at the same concentration equal 16.6 and $66.7 \mu \mathrm{eV}$, respectively. In addition, at $q=0.02 \AA$, the energy width $\Gamma_{2^{\prime} 2^{\prime \prime}}=28 \mu \mathrm{eV}$, if $N_{d 0}=3 \times 10^{12} \mathrm{~cm}^{-2}$, and $\Gamma_{1^{\prime} 1^{\prime \prime}}=100 \mu \mathrm{eV}$, if $N_{d 0}=3 \times 10^{13} \mathrm{~cm}^{-2}$.

The analysis of our calculation results obtained for the dispersion law $\omega^{\prime}(q)$ and the phonon mode width $\Gamma_{i j}\left(i, j=1^{\prime}, 2^{\prime}, \ldots\right)$ of the surface elastic acoustic wave demonstrates (see Figs. 1 and 2) that the phonon mode width $\Gamma_{i j}$ grows in the absence of a non-local interaction (Fig. 2). Furthermore, if the non-local interaction is not taken into consideration $\left(l_{d}=0\right)$, the phase velocity $v_{f}=\frac{\omega(q)}{q}$ of propagation of a quasi-Rayleigh wave is higher in comparison with the case where this interaction is taken into account.

ISSN 2071-0194. Ukr. J. Phys. 2017. Vol. 62, No. 3 
From the boundary conditions (14) and (15) with regard for Eqs. (2) and (3), as well as the orthogonality conditions for the displacement vectors $\mathbf{u}_{n}$ and $\mathbf{u}_{m}(n, m=x, y, z)[20]$

$\frac{1}{a^{3}} \int_{0}^{\frac{1}{k_{l}}} u_{z}^{*}(x, z) u_{z}(x, z) d z=1$,

we obtain the expressions for the amplitudes $|A|$ and $|B|$, which enter the formula for the height of an irregularity created by a quasi-Rayleigh wave and adsorbed atoms on the surface $z=0$. The irregularity height $\tilde{\varsigma}$ is defined as the sum of the normal components of the vectors of displacements for longitudinal $\left(\mathbf{u}^{l}\right)$ and transverse $\left(\mathbf{u}^{t}\right)$ waves at the monocrystal surface $(z=0)$ :

$\tilde{\varsigma}=u_{z}^{l}(0)+u_{z}^{t}(0)$,

where $u_{z}^{l}=k_{l}|A| ; u_{z}^{t}=q|B| ; \frac{1}{k_{l}}$ is the depth of penetration of an acoustic wave into the semiconductor,

$$
\begin{aligned}
& |A|=a^{3 / 2} /\left(\frac{k_{l}}{2}\left(1-\frac{1}{e^{2}}\right)+\frac{k_{l}}{k_{l}+k_{t}} \times\right. \\
& \times \frac{-k_{l}^{2}+(1-2 \beta) q^{2}+M}{\beta k_{t}}\left(1-\frac{1}{e^{1+k_{t} / k_{l}}}\right)+ \\
& +\frac{q^{2}}{2 k_{t}} \frac{\left(-k_{l}^{2}+(1-2 \beta) q^{2}+M\right)^{2}+Z^{2}}{\left(2 \beta k_{t} q\right)^{2}} \times \\
& \times\left(1-\frac{1}{\left.\left.e^{2 k_{t} / k_{l}}\right)\right)^{1 / 2},}\right. \\
& |B|=\sqrt{\frac{\left(-k_{l}^{2}+(1-2 \beta) q^{2}+M\right)^{2}+Z^{2}}{\left(2 \beta k_{t} q\right)^{2}}} a^{3 / 2} / \\
& /\left(\frac{k_{l}}{2}\left(1-\frac{1}{e^{2}}\right)+\frac{k_{l}}{k_{l}+k_{t}} \frac{-k_{l}^{2}+(1-2 \beta) q^{2}+M}{\beta k_{t}} \times\right. \\
& \times\left(1-\frac{1}{e^{1+k_{t} / k_{l}}}\right)+ \\
& +\frac{q^{2}}{2 k_{t}} \frac{\left(-k_{l}^{2}+(1-2 \beta) q^{2}+M\right)^{2}+Z^{2}}{\left(2 \beta k_{t} q\right)^{2}} \times \\
& \left.\times\left(1-\frac{1}{e^{2 k_{t} / k_{l}}}\right)\right)^{1 / 2},
\end{aligned}
$$

where

$$
\begin{aligned}
& M=-\frac{\omega^{\prime 2}}{c_{l}^{2}} \frac{\theta_{d}^{2}}{\rho c_{l}^{2} a} \frac{D_{d}^{2} N_{d 0}}{D_{d}^{2} q^{4}+\omega^{\prime 2}} \frac{q^{2}}{k_{\mathrm{B}} T}\left(1-l_{d}^{2} q^{2}\right), \\
& Z=-\frac{\omega^{\prime 3}}{c_{l}^{2}} \frac{\theta_{d}^{2}}{\rho c_{l}^{2} a} \frac{D_{d} N_{d 0}}{D_{d}^{2} q^{4}+\omega^{\prime 2}} \frac{q^{2}}{k_{\mathrm{B}} T}\left(1-l_{d}^{2} q^{2}\right) .
\end{aligned}
$$

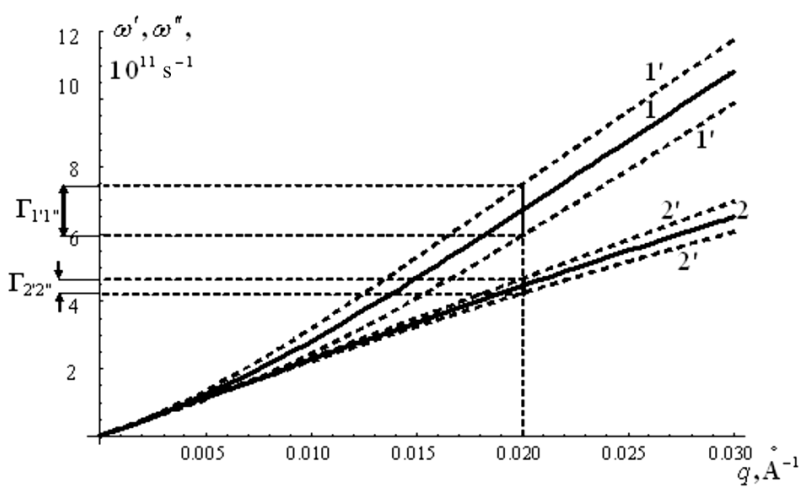

Fig. 2. The same as in Fig. 1, but neglecting the non-local interaction between the adsorbed atom and the matrix atoms $\left(l_{d}=0\right)$

Those amplitudes enter the formula for the height of an irregularity created by a quasi-Rayleigh wave and adsorbed atoms on the surface $z=0$. The irregularity height $\tilde{\varsigma}$ is determined as the sum of the normal components of the displacement vectors for longitudinal $\left(\mathbf{u}^{l}\right)$ and transverse $\left(\mathbf{u}^{t}\right)$ waves at the single-crystal surface $(z=0)$ :

$\tilde{\varsigma}=u_{z}^{l}(0)+u_{z}^{t}(0)$,

where $u_{z}^{l}=k_{l}|A|, u_{z}^{t}=q|B|$, and $\frac{1}{k_{l}}$ is the penetration depth of an acoustic wave into the semiconductor.

\section{Conclusions}

1. A theory describing the dispersion of surface elastic acoustic waves and its dependence on the concentration of adsorbed atoms and the deformation potential has been developed. The theory involves the non-local elastic interaction between the adsorbed atoms and the matrix atoms, as well as image forces.

2 . The energy width of the surface acoustic mode is found to be proportional to the product of the surface concentration of adsorbed atoms and the deformation potential of an adsorbed atom $\left(\Gamma_{i j} \sim N_{d 0} \theta_{d}\right)$.

3. The non-local elastic interaction between the adsorbed atom and the matrix atoms is found to result in a reduction of the energy width of the surface elastic acoustic mode. In the short-wave interval of the acoustic mode, the non-local elastic interaction affects more strongly the variation of the energy width of the surface acoustic elastic mode. 
4. Provided an identical concentration of adsorbed atoms in the near-surface region of the crystal lattice, the width of the surface acoustic mode is found to be larger, if those atoms are interstitial impurities in comparison with the case where they are substitutional impurities. This difference is associated with the fact that the surface deformation potential of adsorbed atoms in the former case (interstitial impurities), $\theta_{d}^{(\mathrm{i})}=K a^{3}$, is larger than in the case where the adsorbed atoms are substitutional impurities, $\theta_{d}^{(\mathrm{s})}=\frac{4}{3} \pi\left(R_{a}^{3}-R_{0}^{3}\right)$, where $R_{a}$ and $R_{0}$ are the covalent-ionic radii of the adsorbed and matrix atoms, respectively.

1. A. Wixforth, J.P. Kotthaus, G. Weimann. Quantum oscillations in the surface-acoustic-wave attenuation caused by a two-dimensional electron system. Phys. Rev. Lett. 56, 2104 (1986) [DOI: 10.1103/PhysRevLett.56.2104].

2. R.L. Willett, K.W. West, L.N. Pfeiffer. Apparent inconsistency of observed composite fermion geometric resonances and measured effective mass. Phys. Rev. Lett. 75, 2988 (1995) [DOI: 10.1103/PhysRevLett.75.2988].

3. D.V. Fil'. Interaction of non-uniform elastic waves with two-dimensional electrons in AlGaAs-GaAs-AlGaAs heterostructures. Low Temp. Phys. 25, 466 (1999) [DOI: 10.1063/1.593768].

4. I.A. Karpovich, A.V. Anshon, D.O. Filatov. Formation and passivation of defects in heterostructures with strained GaAs/InGaAs quantum wells under processing in hydrogen plasma. Fiz. Tekh. Poluprovodn. 32, 1089 (1998) (in Russian).

5. T.W. Kim, H.L. Park. Interband transition and electronic subband studies in CdTeZnTe strained single and double quantum wells grown by double-well temperature-gradient vapor deposition. J. Cryst. Growth 159, 467 (1996) [DOI: 10.1016/0022-0248(95)00636-2].

6. V.I. Kozlovsky, V.G. Litvinov, Yu.G. Sadofyev. Band offset in $\mathrm{Zn}_{1-x} \mathrm{Cd}_{x} \mathrm{Te} / \mathrm{ZnTe}$ single quantum well structure grown by molecular beam epitaxy on GaAs (001). Fiz. Tekh. Poluprovodn. 34, 998 (2000).

7. S.I. Khankina, V.M. Yakovenko, I.V. Yakovenko. Surface electron states produced by a Rayleigh wave. J. Exp. Theor. Phys. 104, 467 (2007) [DOI: 10.1134/ S1063776107030132].

8. M.Ya. Seneta, O.R. Peleshchak, R.M. Peleshchak, Yu.O. Uhryn. In Proceedings of the IX International Conference on Topical Problems of Semiconductor Physics, Truskavets, 2016, edited by R. Pazyuk ("UKRPOL", 2016), p. 152 .

9. V.M. Kovalev, A.V. Chaplik. Interaction of surface and bulk acoustic waves with a two-dimensional semimetal. Zh. Èksp. Teor. Fiz. 147, 356 (2015) [DOI: 10.7868/ S0044451015020145] (in Russian).
10. V.V. Kosachev, Yu.N. Gandurin, S.E. Murav'yov. Influence of a structurally damaged surface layer in an isotropic solid on the dispersion and damping of Rayleigh waves. Fiz. Tverd. Tela 53, 2064 (2011).

11. A.I. Vlasenko, A. Baidullaeva, V.P. Veleschuk, P.E. Mozol, N.I. Boiko, O.S. Litvin. On the formation of nanostructures on a CdTe surface, stimulated by surface acoustic waves under nanosecond laser irradiation. Semiconductors 49, 229 (2015) [DOI: 10.1134/S1063782615020220].

12. R.M. Peleshchak, O.V. Kuzyk, O.O. Dan'kiv. Temperature regimes of formation of nanometer periodic structure of adsorbed atoms in GaAs semiconductors under the action of laser irradiation. Condens. Mat. Phys. 18, 43801 (2015) [DOI: 10.5488/CMP.18.43801].

13. R.M. Peleshchak, I.I. Lazurchak, O.V. Kuzyk, O.O. Dan'kiv, G.G. Zegrya. Role of acoustoelectric interaction in the formation of nanoscale periodic structures of adsorbed atoms. Semiconductors 50, 314 (2016) [DOI: 10.1134/ S1063782616030180].

14. L.D. Landau, E.M. Lifshitz. Theory of elasticity (Pergamon Press, 1970) [ISBN: 8181477928].

15. H. Haken. Synergetik - Eine Einführung (Springer, 1982) [ISBN: 978-3-662-10186-5].

16. I.A. Kunin. Nonlocal Theory of Elasticity (Polish Academy of Sciences, 1970).

17. M.Ya. Krivoglaz. X-ray and Neutron Diffraction in Nonideal Crystals (Springer, 1996) [ISBN: 978-3-642-74291-0].

18. G.L. Falko, S.G. Shpyrko. Model of the deformationinduced redistribution and the stationary concentration profiles of implanted impurity. Ukr. Fiz. Zh. 40, 1115 (1995) (in Ukrainian).

19. V.I. Emel'yanov, K.I. Eremin. Threshold nucleation of a nanometer-scale periodic adatom structure with the participation of a static surface acoustic wave. JETP Letters 75, 98 (2002) [DOI:10.1134/1.1466485].

20. N. Bannov, V. Mitin, M. Stroscio. Confined acoustic phonons in a free-standing quantum well and their interaction with electrons. Phys. Status Solidi B 183, 131 (1994) [DOI: 10.1002/pssb.2221830109].

Received 18.11.16.

Translated from Ukrainian by O.I. Voitenko

Р.М. Пелещак, М.Я. Сенета

ЗАКОН ДИСПЕРСІЇ ТА ШИРИНА

ПОВЕРХНЕВОЇ АКУСТИЧНОЇ МОДИ

В ЗАЛЕЖНОСТІ ВІД КОНЦЕНТРАЦЇ̈

АДСОРБОВАНИХ АТОМІВ

$\mathrm{P}$ е $з$ ю м е

У межах моделі взаємодії адатомів з самоузгодженою акустичною квазірелеєвською хвилею в довгохвильовому наближенні з урахуванням сил дзеркального зображення та нелокальної пружної взаємодії адсорбованого атома з атомами матриці знайдено закон дисперсії поверхневих пружних акустичних хвиль і ширину поверхневої акустичної моди в залежності від концентрації адсорбованих атомів. 